\title{
Predictors of Mortality in Pediatric Patients on Venoarterial Extracorporeal Membrane Oxygenation
}

\author{
Rajesh Punn, MD, David M. Axelrod, MD, Sara Sherman-Levine, RN, MSN, CPNP, Stephen \\ J. Roth, MD, MPH, and Theresa A. Tacy, MD \\ Division of Pediatric Cardiology, Department of Pediatrics, Stanford University School of \\ Medicine, Lucile Packard Children's Hospital
}

\section{Abstract}

Background-Currently, there are no established echocardiographic (echo) or hemodynamic predictors of mortality after weaning venoarterial extracorporeal membrane oxygenation (ECMO) in children. We wished to determine which measurements predict mortality.

Methods-Over 3 years, we prospectively assessed six echo and six hemodynamic variables at 3-5 circuit rates while weaning ECMO flow. Hemodynamic measurements were: heart rate, inotropic score, arterio-venous oxygen difference (AV-O2), pulse pressure, oxygenation index (OI), and lactate. Echo variables included: shortening/ejection fraction, outflow tract Dopplerderived stroke distance (VTI), degree of atrioventricular valve regurgitation, longitudinal strain (GLS), and circumferential strain (GCS). Patients were stratified into those who died or required heart transplant (Gr1), and those who did not (Gr2). For each patient, we compared the change for each variable between full versus minimum ECMO flow for each group.

Results-We enrolled 21 patients ranging in age 0.02-15 years. Five had dilated cardiomyopathy, and 16 had structural heart disease with severe ventricular dysfunction. Thirteen of 21 patients (62\%) comprised Gr1, including two patients with heart transplants. Eight patients constituted Gr2. Gr1 patients had a significantly greater increase in OI (35\% mean increase, $\mathrm{p}<0.01$ ) off ECMO compared to full flow, but no change in VTI or AV-O2. In Gr2 VTI increased ( $31 \%$ mean increase, $\mathrm{p}<0.01$ ) with no change in AV-O2 or OI. Pulse pressure increased modestly with flow reduction only in $\mathrm{Gr} 1(\mathrm{p}<0.01)$.

Conclusion-Failure to augment VTI or an increase in OI during the ECMO weaning is associated with poor outcomes in children. We propose that these measurements should be performed during ECMO wean, as they may discriminate who will require alternative methods of circulatory support for survival.

\section{Keywords}

extracorporeal membrane oxygenation; echocardiography; surgery

Address for reprints: Rajesh Punn, MD, Division of Pediatric Cardiology, 750 Welch Road, Suite \# 325, Palo Alto, CA 94304, Tel: (650) 721-6720, Fax: (650) 724-4922, rpunn@stanford.edu.

Disclosures: None 


\section{Introduction}

Venoarterial extracorporeal membrane oxygenation (ECMO) is periodically used to provide temporary mechanical circulatory support in children with cardiomyopathy or postoperative ventricular dysfunction who have potential for recovery of cardiac function. The mortality rate for cardiac patients undergoing such mechanical support is $40-60 \%$ (1-7). The risk factors for mortality in children undergoing ECMO support are numerous, and include renal failure, length of time on mechanical support, and single ventricle physiology $(1,2,8-11)$. However, if a patient is determined to have a low chance of recovery of ventricular function on ECMO when reassessed, ventricular assist device (VAD) support may be contemplated as a bridge to orthotopic heart transplantation (12).

Echocardiography (echo) can accurately identify cannula position, detect thrombus formation and pericardial effusions, and guide left atrial decompression (13-16). However, echo is frequently utilized to assess the ventricular function of a patient on ECMO, when preload is decreased and afterload is increased (17-21), situations which complicate the assessment of myocardial function using conventional echocardiographic measures. Presumably, severely depressed ventricular systolic function leading to inadequate cardiac output increases the mortality rate and need for heart transplant. The purpose of our study was to identify prospectively echocardiographic and hemodynamic predictors of mortality in patients on ECMO for cardiomyopathy or postoperative ventricular dysfunction during weaning of ECMO flow.

\section{Methods}

\section{Patient population}

From January 2010 to December 2012, all patients receiving venoarterial ECMO support in the cardiovascular intensive care unit at our institution were enrolled in this study. The patients' date of birth, date of discharge or death, and duration of ECMO support were recorded. Patients who died or needed a heart transplant were defined as the organ failure group (Gr1), while the remaining patients were defined as the organ survival group (Gr2; alive and not transplanted before hospital discharge). All patients were consented by a research coordinator (SS). This study is supported in part by NIH-NCATS-CTSA grant \#UL1 TR001085, Lucile Packard Foundation for Children's Health, and the Child Health Research Institute. The Stanford Institutional Review Board approved the study.

\section{ECMO circuit}

Indications for ECMO support included: myocarditis, tachycardia-induced cardiomyopathy, cardiac arrest after cardiac surgery for congenital heart disease, or failure to wean from cardiopulmonary bypass during surgery. After at least 48 hours of ECMO support, the cardiac intensivist managing the patient decided the timing of weaning based on systemic arterial pulsatility, clinical and biochemical markers of appropriate end-organ perfusion, and stable clinical status. Patients were supported with $100-130 \mathrm{ml} / \mathrm{kg} / \mathrm{min}$ of ECMO flow, which for study purposes we defined as full flow. Patients with patent aortopulmonary shunts received higher flows; their mean flow rate was $120 \mathrm{ml} / \mathrm{kg} / \mathrm{min}$. The ECMO circuit 
pump utilized at our institution is either a Sorin S3 or Sorin Centrifugal "Revolution."

During the ECMO flow weaning process, an attending pediatric echocardiographer (TT/RP), attending cardiac intensivist managing the patient, and a sonographer were present. The intensivist directed the flow rates during the ECMO flow wean. Steady-state hemodynamics were obtained by waiting for at least five minutes at each specific ECMO flow rate prior to any data collection. Each wean increment was between $10-30 \mathrm{ml} / \mathrm{kg} / \mathrm{min}$. The hemodynamic information and echo data were collected at 3-5 flow rates for each patient by one of two echocardiography attendings (TT/RP) since it was unclear what the lowest tolerated ECMO flow rate would be. Each patient had zero to minimal flow as a targeted final setting; minimal flow was defined as less than $20 \mathrm{ml} / \mathrm{kg} / \mathrm{min}$. The cardiothoracic surgeon and managing intensivist determined each patient's eligibility for ECMO decannulation.

\section{Hemodynamics}

For each ECMO flow rate reduction, the patient's heart rate, heart rhythm, blood pressure, arterial/venous blood gas analysis, mean airway pressure, inspired oxygen content, lactate level, and inotropic support were recorded. From these data, we derived an arterio-venous oxygen saturation difference (AV-O2), oxygenation index (OI) (22), and revised inotrope score(23). The formula for oxygenation index is:

$$
\mathrm{P} \overline{\mathrm{aw}} \times \mathrm{FiO} 2 \times 100 / \mathrm{PaO} 2,
$$

where $\mathrm{P} \overline{\mathrm{aw}}$ is the mean airway pressure, $\mathrm{FiO} 2$ is the inspired oxygen content, and $\mathrm{PaO} 2$ is the arterial partial pressure of oxygen.

For those patients with an atrial stent or a left atrial vent cannula, accurate mixed venous oxygen saturation could not be obtained, and thus the arterio-venous oxygen saturation could not be calculated. The blood gas analyses were obtained from a point-of-care I-stat ${ }^{\circledR}$ machine at the bedside (Abbott Laboratories, Abbott Park, Illinois).

\section{Echocardiography}

All echocardiograms were performed on the Siemens Sequoia C512, rev 12.0 (Mountain View, California). Images were recorded with acoustic capture at high frame rates from the parasternal long and short axis and apical four- and five-chamber views; the average frame rate measured between $50-60 \mathrm{~Hz}$ for pre- and post-wean studies. At least 9 images ( 3 separate captures of 3-beat intervals) were obtained optimizing image quality and minimizing lung artifact. An echocardiographer (TT or RP) was present at each image acquisition to ensure the best possible image quality. M-mode imaging and apical imaging were utilized to determine a shortening fraction (FS) and ejection fraction (EF) per American Society of Echocardiography guidelines (24). A color Doppler image of the systemic atrioventricular valve was obtained and one of the readers (RP) provided a qualitative assessment of the degree of regurgitation. The degree of atrioventricular valve regurgitation was categorized on a 0 to 4 scale (25). Pulsed-wave Doppler acquisition of the systemic ventricular outflow tract was obtained and a stroke distance, velocity-time-integral (VTI) in meters, was obtained by measuring the area under the curve. A minimum of 9 
complete pulsed-wave Doppler signals were obtained, and the highest velocity signals were averaged to minimize the effect of respiration on preload. We focused on ensuring that the Doppler angles were aligned with flow, and that the angle of insonation was consistent throughout each patient's echocardiogram at each ECMO flow rate. Offline speckle tracking analysis was performed with Velocity Vector Imaging 2.0 (Siemens Medical Solutions USA, Inc, Syngo Dynamics Solutions) to determine the global longitudinal strain (GLS), global longitudinal strain rate (GLSR), global circumferential strain (GCS) and global circumferential strain rate (GCSR). The strain and strain rate measurements were obtained over three cardiac cycles and averaged. As in our prior work, the peak of the average curve was used for each cycle. Of note, some inter- and intraobserver variability was noted in our laboratory and in other studies (26-29). All data were obtained at 3 to 5 ECMO flow rates, including minimal and full flow, which are defined above. The EF and FS were not obtained in single right ventricle patients, given the abnormal geometry that accompanies these ventricular shapes; however, GLS and GLSR were determined for these patients.

\section{Statistics}

All collected data were tested for normalcy using a Kolmogorov-Smirnov test. Variables were expressed as either a mean and standard deviation or median with range. Full and minimal flow data points were compared using a paired T test or a Wilcoxon Signed Rank test, accordingly. Hemodynamic and echocardiographic data of patients stratified as having organ death (Gr1) and no organ death (Gr2) were compared using an unpaired Student's T test or a Mann-Whitney U test. Fisher's exact tests were used to compare the percentage of patients with either single ventricle or shunt-dependent physiology, the presence or absence of congenital heart disease, and type of cannulation site in each of these groups. To avoid making a type 1 error, a post-Bonferroni correction (B-corrected) was applied for those data that were initially deemed statistically significant by multiplying the number of variables (12) by the $p$-value. Only a $p$-value less than 0.05 after this correction was considered statistically significant. All statistical testing was conducted using SAS Enterprise Guide 4.3 (SAS Institute Inc, Cary, North Carolina).

\section{Results}

\section{Patient population}

Twenty-one patients were enrolled and underwent 24 weaning attempts. One patient had two wean attempts and another had three attempts. Patients with recent surgery and on ECMO for less than 48 hours did not undergo ECMO flow weaning with concurrent echocardiographic evaluation and were excluded from the study. Two consented patients underwent a weaning attempt when the echocardiographers participating in the study (RP/TT) were unavailable and were thus excluded from analysis. An additional three patients had no pulsatility on ECMO, so a wean attempt was not undertaken. Two of these patients died and were then decannulated while the third was transitioned to a ventricular assist device and had a subsequent heart transplant.

The initial wean attempt was used for analysis, and all patients had one ECMO course. To prevent aortopulmonary shunt occlusion or ECMO circuit thrombus formation, some 
patients' minimum flow was only decreased to $40 \mathrm{ml} / \mathrm{kg} / \mathrm{min}$. The average time for ECMO wean and echocardiogram study was $58.7 \pm 37.3$ minutes. The reason for ECMO was severely decreased ventricular function in all patients, of which three patients had associated ventricular arrhythmias and one patient failed to separate from cardiopulmonary bypass in the operating room. An occluded aortopulmonary shunt for pulmonary blood flow and pulmonary arterial hypertension were not reasons for ECMO initiation in any of the patients included in the study. The patients' median age was 2.4 years (range 0.02 to 15.5 ) with varying cardiac diagnoses (Table 1). Eleven patients (52\%) died during hospital admission and two (10\%) received an orthotopic heart transplant. Thus, organ failure, defined as death or heart transplant, occurred in a total of 13 patients (62\%); these 13 patients comprise Gr1. Both heart transplant patients were transitioned to biventricular assist devices as a bridge to transplant. One was transitioned directly from ECMO, while the other was decannulated for 11 days and then transitioned. Five patients who died were decannulated between 1-9 days prior to death. One subject died 90 days after decannulation, while five others died at the time of decannulation. All deaths were either due to sudden cardiac arrest or multiorgan failure.

In $\mathrm{Gr} 1,85 \%$ patients had congenital heart disease while in $\mathrm{Gr} 275 \%$ of patients had structurally normal hearts; however, this difference did not achieve statistical significance $(p$ $=0.0176$, B-corrected $p=0.21$ ). A left atrial cannula was positioned or an interatrial communication was created in six patients, while four patients had a natural atrial communication with left-to-right shunting. Thus, an AV-O2 was not obtained in these ten patients. Sites for ECMO cannula insertion included: neck (14), intrathoracic (6), and femoral (1). The percentage of intrathoracic cannulation was not higher than neck in Gr1 versus $\mathrm{Gr} 2(31 \%$ vs $29 \%, p=1.00)$. The mean ECMO flow rates were $97 \mathrm{ml} / \mathrm{kg} / \mathrm{min}$ and 38 $\mathrm{ml} / \mathrm{kg} / \mathrm{min}$ at full and minimal flow, respectively. Five patients had aortopulmonary shunts or circuits that required more flow and prevented lower ECMO flow rates, while three patients were weaned to below $20 \mathrm{ml} / \mathrm{kg} / \mathrm{min}$. Thirteen patients were clamped and received no ECMO flow during the wean process. The median length of ECMO support was 264 hours (range 96 to 1200) for Gr1 versus 132 hours (range 96 to 264) for $\mathrm{Gr} 2$ ( $p=0.04$, Bcorrected $p=0.48$ ).

\section{Hemodynamic data}

Each patient's pulse pressure, AV-O2, and OI increased as ECMO flow was reduced from full to minimal or no flow (Table $1, p=0.0005, p=0.0031$, and $p=0.0051$, respectively). The OI did not retain significance with Bonferroni correction (B-corrected $p=0.06$ ). In contrast, the heart rate, inotrope score, and serum lactate did not change under these varying conditions (Table 1). When stratifying Gr1 and Gr2, pulse pressure increased modestly in both groups upon decreasing to minimal ECMO flow; however, only Gr1 achieved statistical significance (Table 2, $p=0.0025$, B-corrected $p=0.03$ ). AV-O2, on the other hand, showed a trend towards a difference in $\operatorname{Gr} 1(p=0.0062$, B-corrected $p=0.07)$, while $\mathrm{Gr} 2$ had no change in the AV-O2 when weaning from full to minimal flow $(p=0.48)$.

As flow was weaned, OI was not statistically different in $\operatorname{Gr} 2(p=0.12)$, but patients in Gr1 had a significant increase in OI $(p=0.0023$, B-corrected $p=0.03)$. The percentage of single 
ventricle patients or shunt-dependent patients were higher in $\mathrm{Gr} 1(31 \%)$ compared to $\mathrm{Gr} 2$ $(13 \%)$. However, given the small sample size, this difference in the percentage of single ventricles patients was not statistically significant $(p=0.28)$. A subanalysis of OI in $\mathrm{Gr} 1$ excluding patients with single ventricle or shunt-dependent patients continued to show a significant increase in OI $(p=0.02)$.

The age ( $p=0.27)$ and heart rate $(p=0.25)$ were no different between groups. Of note, the absolute measurement of OI on full ECMO flow was no different between groups (7.5 \pm 9.6 for $\mathrm{Gr} 1$ and $5.9 \pm 3.9$ for $\mathrm{Gr} 2, p=0.56$ ). Also, the AV-O2 at full ECMO flow was also no different between groups after Bonferroni correction (36.1\% for $\mathrm{Gr} 1$ and $23.5 \%$ for $\mathrm{Gr} 2, p$ $=0.0284$, B-corrected $p=0.34$ ).

\section{Echocardiographic data}

None of the echocardiographic measurements including SF, EF, and degree of atrioventricular valve regurgitation changed significantly going from full to minimal or no flow for the entire cohort (Table 2). GLSR was close to being statistically significant ( $p=$ 0.09 ). However, when stratified by organ death, the VTI increased only in Gr2 subjects ( $p=$ 0.0028 , B-corrected $p=0.03$ ) while those in Gr1 had no significant change in VTI ( $p=$ 0.35 ). On average the percent change increase was only $19.6 \%$ for those in Gr1, while the percent change in those without organ death, Gr2, was 31.3\% (Figure 1). Similar to the hemodynamic measurements, the absolute VTI prior to ECMO wean was no different between $\mathrm{Gr} 1$ and $\mathrm{Gr} 2(0.04 \pm 0.03$ vs $0.06 \pm 0.02$ meters, $p=0.35)$.

\section{Discussion}

We believe that our prospective study is the first to establish hemodynamic and echocardiographic measurements that predict mortality in pediatric cardiac patients supported with venoarterial ECMO. Specifically, when weaning ECMO flow from full to minimal/no flow, those with subsequent organ death (Gr1) had no significant augmentation in VTI and a significant increase in OI compared to those patients without organ death (Gr2). The VTI, a surrogate of cardiac output, is a readily-available, non-invasive measurement, is not influenced by atrioventricular valve regurgitation or abnormal ventricular geometry. VTI varies with age and body size; in this study, each patient served as his/her own comparison. The percentage change in VTI as ECMO flow was weaned was a predictor of post-ECMO organ survival. Based on these data, we advocate obtaining this measurement on all ECMO patients undergoing an echocardiogram during ECMO flow weaning. Of note, when comparing the absolute values of prewean VTI, OI, and AV-O2 between the two groups, there was no difference, emphasizing that the weaning process is necessary to discern outcomes in these patients.

Only one study by Aissaoui et al. has investigated VTI in this setting, and they discovered that it was higher $(>0.10 \mathrm{~m})$ in adults who eventually weaned, but the authors did not discuss the prediction of mortality using VTI (30). An absolute VTI measurement cannot be applied in the pediatric population given the changes in VTI over age and body size. No pediatric study has investigated this method of establishing cardiac output in the ECMO setting. Like all of the echocardiographic assessments of ventricular performance, VTI is also load- 
dependent, which explains the increase noted with ECMO flow weaning and increasing preload. Given the nature of Doppler-derived measurements, accurate VTI assessment is angle-dependent, and we highly recommend VTI comparisons have a minimal angle of insonation at all measurements.

OI has been used to establish the need for ECMO in neonates with persistent pulmonary hypertension and is an excellent marker for the degree of difficulty with oxygenation (22); a measurement over 40 predicted a need for ECMO, while an OI less than 35 predicted survival in another study (4). In our study, those with organ death had a significant increase in the OI after ECMO was weaned compared to those without organ death. Of note, a confounding variable is the relatively higher percentage of patients with single ventricle physiology and shunt dependency for pulmonary blood flow. Excluding these patients, however, availed the same increase in OI in the subgroup with adverse outcome. Given the clinical implications, OI may have some applications to those on ECMO. AV-O2 changes with ECMO wean, on the other hand, did not quite reach statistical significance and a subset of patients have atrial-level shunts (either native or created atrial communications) which preclude an accurate measure of mixed venous oxygen saturation.

As noted in other studies, length of ECMO support was longer in the organ death group (Gr1) of our study; however, this did not achieve statistical significance $(1,9,10)$. The risks of prolonged ECMO support with associated morbidity and mortality from complications over time have been well described. Cannulation site and presence of congenital heart disease had no bearing on outcome in our 21 patients. Depressed GLS, GLSR, GCS, and GCSR measurements at full flow were also not associated with poor outcome in our study. The image quality, though optimized to the best of our ability, was often somewhat limited by the patient position, poor acoustic windows from chest tube placement, and/or the presence of an open sternum. Thus, frame rate and acoustic image quality may have influenced our ability to use deformation techniques, which in prior studies can detect subtle myocardial dysfunction (26,31-33).

Many of our predictors were not significantly different upon weaning ECMO flow, which may be due to our small sample size. Since VTI is independent of geometry and is an assessment of cardiac output, it is an ideal way to monitor ventricular performance during loading changes that are expected to alter geometry and cardiac size. Other studies have investigated ventricular performance while on ECMO with variable results (17-20). Meridional or circumferential wall stress, a marker of ventricular afterload, has been shown to increase on ECMO support, while velocity of circumferential fiber shortening ( $\mathrm{VCFc}$ ) and FS, measures of ventricular function, have been shown to decrease $(17,18)$.

Gr1 patients had an increase in pulse pressure compared to Gr2 with ECMO wean; however, the magnitude of this difference is probably not clinically relevant. Some also argue that simply inserting a relatively large cannula in the aorta increases afterload as well. Other studies show no change in the wall stress or VCFc on ECMO support (19). Almost all of these studies were performed on neonates with pulmonary hypertension, while our cohort included older patients with a primary myocardial indication for the ECMO support. In these prior studies, the indications were for pulmonary hypertension and not decreased ventricular 
function. One of the studies was performed on sheep, and it demonstrated a decrease in wall stress for normal sheep hearts on ECMO and an increase in wall stress for ischemia-induced diseased hearts (18). It is unclear how wall stress is altered in children on ECMO for cardiac failure. Studies of wall stress on ECMO patients with myocardial dysfunction need to be conducted to determine the impact of ECMO on afterload. To minimize the increased myocardial oxygen demand from these altered loading conditions, we would propose earlier weaning using the OI and VTI as supplemental information to the other clinical data readily available earlier in the process. Failure of adequate output augmentation during ECMO weaning using these criteria may assist with consideration for alternate long-term circulatory support as a bridge to transplantation.

\section{Limitations}

Our study, while prospective, has a small sample size and was performed at a single institution. Some of the hemodynamic and echocardiographic variables may prove to significant predictors of adverse outcome with a larger sample size. As a result, we have embarked on a larger, multi-center investigation to increase the sample size of our study. Our study did demonstrate that VTI should be considered as one element of assessing cardiac performance during an ECMO wean. We acknowledge that confirmation of our initial findings will be needed prior to development of stronger recommendations for the management of these challenging patients.

\section{Conclusion}

Failure to augment VTI adequately during ECMO flow reduction or an increase in OI is associated with poor outcomes in pediatric patients. VTI should be included as a part of a systemic approach to the assessment of ventricular performance during ECMO wean. Future studies are indicated to confirm these findings with a larger sample size.

\section{References}

1. Kolovos NS, Bratton SL, Moler FW, et al. Outcome of pediatric patients treated with extracorporeal life support after cardiac surgery. Ann Thorac Surg. 2003; 76:1435-41. discussion 1441-2. [PubMed: 14602263]

2. Morris MC, Ittenbach RF, Godinez RI, et al. Risk factors for mortality in 137 pediatric cardiac intensive care unit patients managed with extracorporeal membrane oxygenation. Crit Care Med. 2004; 32:1061-9. [PubMed: 15071402]

3. Derby CD, Kolcz J, Kerins PJ, Duncan DR, Quezada E, Pizarro C. Aristotle score predicts outcome in patients requiring extracorporeal circulatory support following repair of congenital heart disease. ASAIO J. 2007; 53:82-6. [PubMed: 17237653]

4. Mehta NM, Turner D, Walsh B, et al. Factors associated with survival in pediatric extracorporeal membrane oxygenation--a single-center experience. J Pediatr Surg. 2010; 45:1995-2003. [PubMed: 20920718]

5. Delmo Walter EM, Alexi-Meskishvili V, Huebler M, et al. Extracorporeal membrane oxygenation for intraoperative cardiac support in children with congenital heart disease. Interact Cardiovasc Thorac Surg. 2010; 10:753-8. [PubMed: 20139198]

6. Chauhan S, Malik M, Malik V, Chauhan Y, Kiran U, Bisoi AK. Extra corporeal membrane oxygenation after pediatric cardiac surgery: a 10 year experience. Ann Card Anaesth. 2011; 14:1924. [PubMed: 21196670] 
7. Skinner SC, Iocono JA, Ballard HO, et al. Improved survival in venovenous vs venoarterial extracorporeal membrane oxygenation for pediatric noncardiac sepsis patients: a study of the Extracorporeal Life Support Organization registry. J Pediatr Surg. 2012; 47:63-7. [PubMed: 22244394]

8. Rajagopal SK, Almond CS, Laussen PC, Rycus PT, Wypij D, Thiagarajan RR. Extracorporeal membrane oxygenation for the support of infants, children, and young adults with acute myocarditis: a review of the Extracorporeal Life Support Organization registry. Crit Care Med. 2010; 38:382-7. [PubMed: 19789437]

9. Kumar TK, Zurakowski D, Dalton H, et al. Extracorporeal membrane oxygenation in postcardiotomy patients: factors influencing outcome. J Thorac Cardiovasc Surg. 2010; 140:330336. e2. [PubMed: 20637917]

10. Rood KL, Teele SA, Barrett CS, et al. Extracorporeal membrane oxygenation support after the Fontan operation. J Thorac Cardiovasc Surg. 2011; 142:504-10. [PubMed: 21353253]

11. Ugaki S, Kasahara S, Kotani Y, et al. Extracorporeal membrane oxygenation following Norwood stage 1 procedures at a single institution. Artif Organs. 2010; 34:898-903. [PubMed: 21092032]

12. Pagani FD, Lynch W, Swaniker F, et al. Extracorporeal life support to left ventricular assist device bridge to heart transplant: A strategy to optimize survival and resource utilization. Circulation. 1999; 100:II206-10. [PubMed: 10567305]

13. Koenig PR, Ralston MA, Kimball TR, Meyer RA, Daniels SR, Schwartz DC. Balloon atrial septostomy for left ventricular decompression in patients receiving extracorporeal membrane oxygenation for myocardial failure. J Pediatr. 1993; 122:S95-9. [PubMed: 8501556]

14. Riccabona M, Dacar D, Zobel G, et al. Sonographically guided cannula positioning for extracorporeal membrane oxygenation. Pediatr Radiol. 1995; 25:643-5. [PubMed: 8570320]

15. Katz WE, Jafar MZ, Mankad S, Keenan RJ, Martich GD. Transesophageal echocardiographic identification of a malpositioned extracorporeal membrane oxygenation cannula. J Heart Lung Transplant. 1995; 14:790-2. [PubMed: 7578192]

16. Moubarak G, Weiss N, Leprince P, Luyt CE. Massive intraventricular thrombus complicating extracorporeal membrane oxygenation support. Can J Cardiol. 2008; 24:e1. [PubMed: 18209764]

17. Martin GR, Short BL. Doppler echocardiographic evaluation of cardiac performance in infants on prolonged extracorporeal membrane oxygenation. Am J Cardiol. 1988; 62:929-34. [PubMed: 3177240]

18. Bavaria JE, Ratcliffe MB, Gupta KB, Wenger RK, Bogen DK, Edmunds LH Jr. Changes in left ventricular systolic wall stress during biventricular circulatory assistance. Ann Thorac Surg. 1988; 45:526-32. [PubMed: 3365043]

19. Kimball TR, Daniels SR, Weiss RG, et al. Changes in cardiac function during extracorporeal membrane oxygenation for persistent pulmonary hypertension in the newborn infant. J Pediatr. 1991; 118:431-6. [PubMed: 1999787]

20. Berdjis F, Takahashi M, Lewis AB. Left ventricular performance in neonates on extracorporeal membrane oxygenation. Pediatr Cardiol. 1992; 13:141-5. [PubMed: 1603713]

21. Tanke RB, Daniels O, van Heijst AF, van Lier H, Festen C. Cardiac dimensions during extracorporeal membrane oxygenation. Cardiol Young. 2005; 15:373-8. [PubMed: 16014184]

22. Durand M, Snyder JR, Gangitano E, Wu PY. Oxygenation index in patients with meconium aspiration: conventional and extracorporeal membrane oxygenation therapy. Crit Care Med. 1990; 18:373-7. [PubMed: 2318047]

23. Gaies MG, Gurney JG, Yen AH, et al. Vasoactive-inotropic score as a predictor of morbidity and mortality in infants after cardiopulmonary bypass. Pediatr Crit Care Med. 2010; 11:234-8. [PubMed: 19794327]

24. Lopez L, Colan SD, Frommelt PC, et al. Recommendations for quantification methods during the performance of a pediatric echocardiogram: a report from the Pediatric Measurements Writing Group of the American Society of Echocardiography Pediatric and Congenital Heart Disease Council. J Am Soc Echocardiogr. 2010; 23:465-95. quiz 576-7. [PubMed: 20451803]

25. Miyatake K, Izumi S, Okamoto M, et al. Semiquantitative grading of severity of mitral regurgitation by real-time two-dimensional Doppler flow imaging technique. J Am Coll Cardiol. 1986; 7:82-8. [PubMed: 3941221] 
26. Lowenthal A, Tacy TA, Behzadian F, Punn R. Echocardiographic Predictors of Early Postsurgical Myocardial Dysfunction in Pediatric Patients With Aortic Valve Insufficiency. Pediatr Cardiol. 2013

27. Singh GK, Cupps B, Pasque M, Woodard PK, Holland MR, Ludomirsky A. Accuracy and reproducibility of strain by speckle tracking in pediatric subjects with normal heart and single ventricular physiology: a two-dimensional speckle-tracking echocardiography and magnetic resonance imaging correlative study. J Am Soc Echocardiogr. 2010; 23:1143-52. [PubMed: 20850945]

28. Sun HY, Behzadian F, Punn R, Tacy TA. Decremental left ventricular deformation after pulmonary artery band training and subsequent repair in ventriculoarterial discordance. J Am Soc Echocardiogr. 2013; 26:765-74. [PubMed: 23623593]

29. Truong UT, Sun HY, Tacy TA. Myocardial deformation in the fetal single ventricle. J Am Soc Echocardiogr. 2013; 26:57-63. [PubMed: 23140844]

30. Aissaoui N, Luyt CE, Leprince P, et al. Predictors of successful extracorporeal membrane oxygenation (ECMO) weaning after assistance for refractory cardiogenic shock. Intensive care medicine. 2011; 37:1738-45. [PubMed: 21965097]

31. Di Salvo G, Pacileo G, Del Giudice EM, et al. Abnormal myocardial deformation properties in obese, non-hypertensive children: an ambulatory blood pressure monitoring, standard echocardiographic, and strain rate imaging study. European Heart Journal. 2006; 27:2689-2695. [PubMed: 16905554]

32. Ganame J, Claus P, Uyttebroeck A, et al. Myocardial Dysfunction Late After Low-Dose Anthracycline Treatment in Asymptomatic Pediatric Patients. Journal of the American Society of Echocardiography : official publication of the American Society of Echocardiography. 2007; 20:1351-1358. [PubMed: 17604960]

33. Khoo NS, Smallhorn JF, Kaneko S, Kutty S, Altamirano L, Tham EB. The Assessment of Atrial Function in Single Ventricle Hearts from Birth to Fontan: A Speckle-Tracking Study by Using Strain and Strain Rate. Journal of the American Society of Echocardiography : official publication of the American Society of Echocardiography. 2013; 26:756-764. [PubMed: 23669597] 


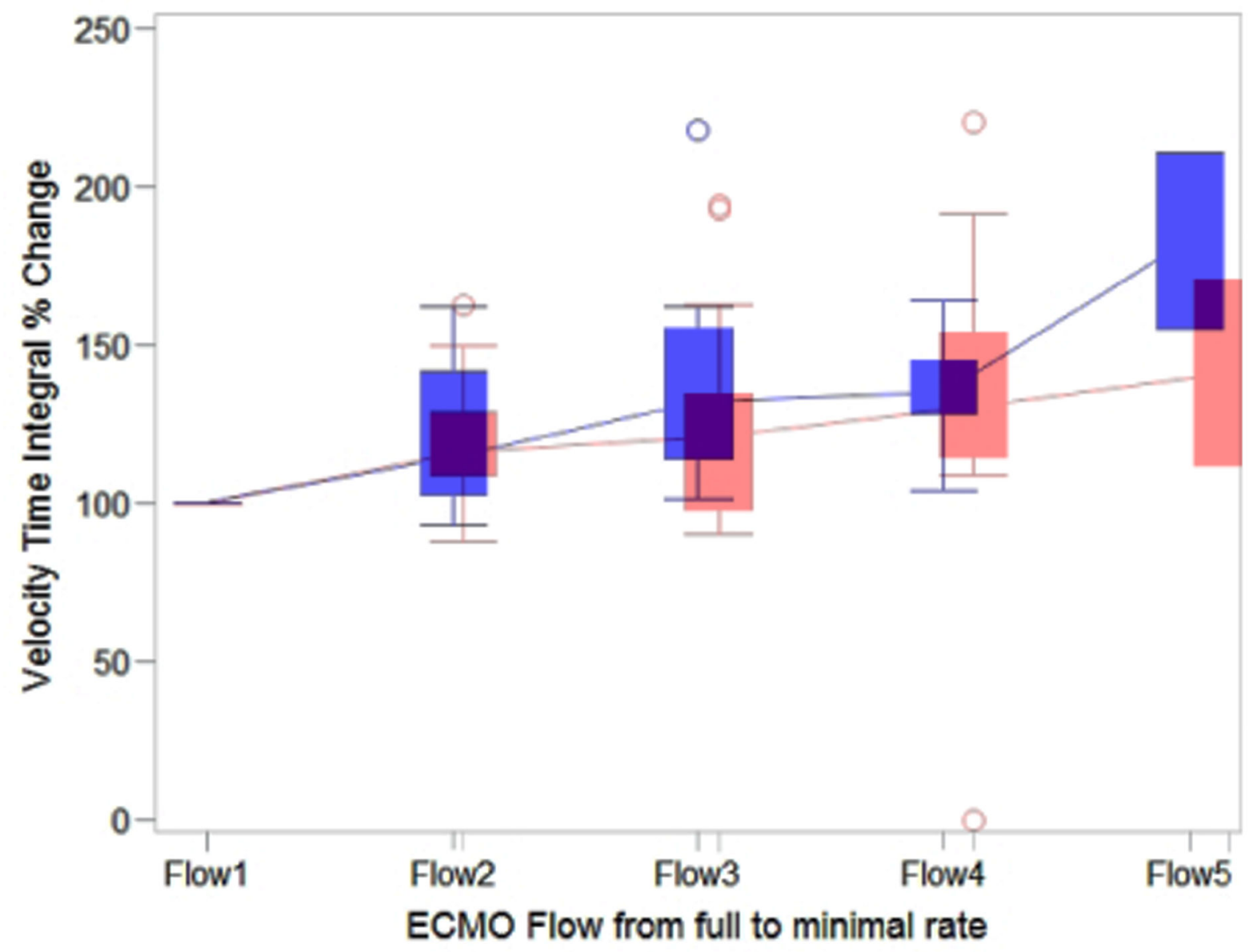

Figure 1.

Box-whisker plot showing the VTI percent change from full flow (flow 1) to minimal flow (flow 3-5) in Group 1 (RED) is shown. There is an overall increase in VTI. However, the percent change in VTI is greater in Group 2 patients (BLUE) upon weaning flow. All patients' flow was decreased from full to minimal flow through four steps. The vertical bars represent 1.5 times the interquartile range. 


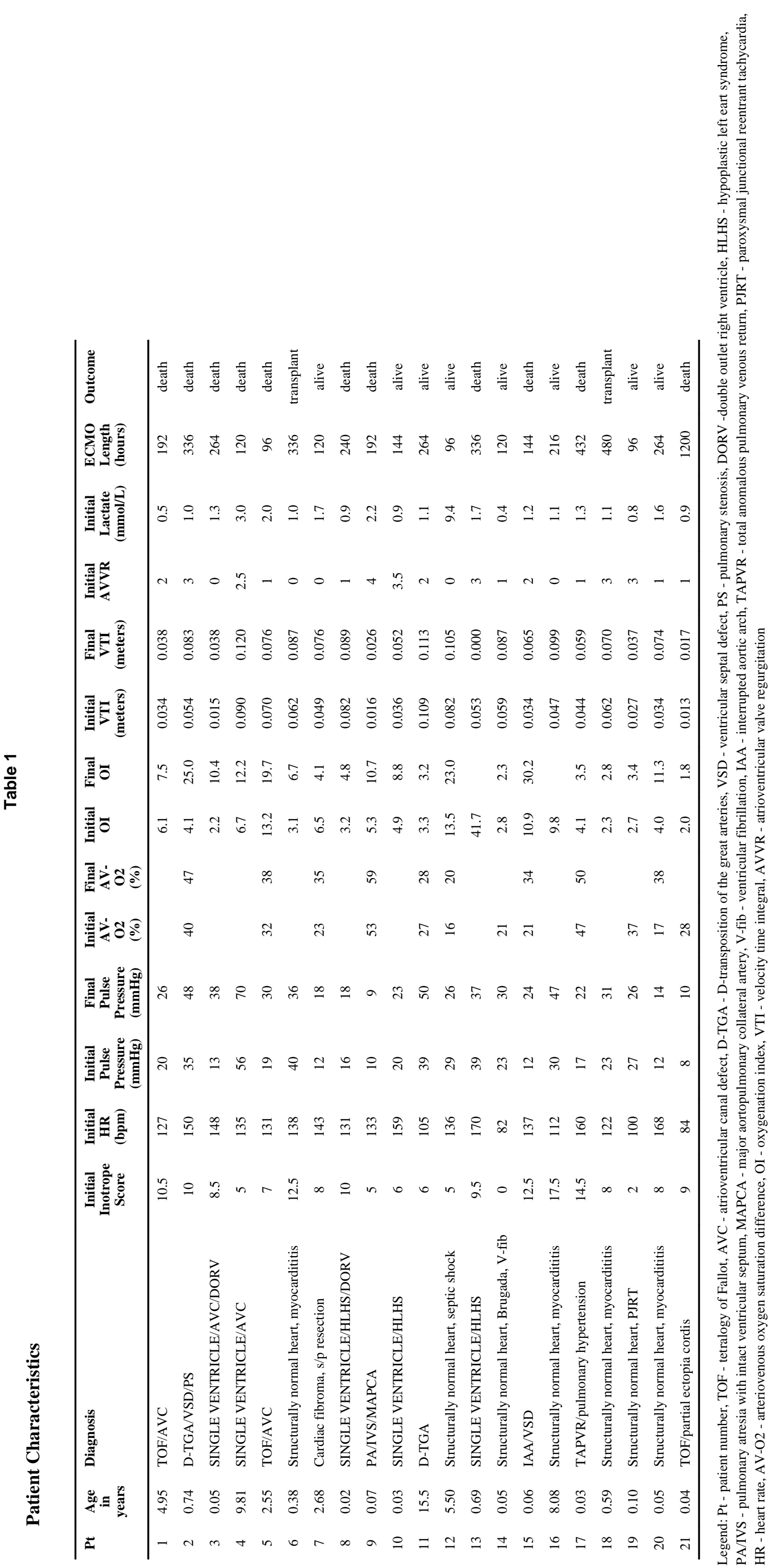


Table 2

\section{Comparison of Group 1 and 2}

\begin{tabular}{|l|c|c|c|}
\hline Variable & Group 1 (n= 13) & Group 2 (n = 8) & $p$-value \\
\hline Initial Heart Rate (bpm) & $137.6 \pm 18.9$ & $125.6 \pm 30.4$ & 0.25 \\
\hline Pulse Pressure Change (mmHg) & $7.1 \pm 14.6$ & $5.3 \pm 11.1$ & $\mathbf{0 . 0 3}, 1.00$ \\
\hline Oxygenation Index Change & $3.9 \pm 8.9$ & $2.1 \pm 5.6$ & $\mathbf{0 . 0 3}, 1.00$ \\
\hline Initial Inotropic score & $10.1 \pm 3.7$ & $6.6 \pm 5.2$ & 0.12 \\
\hline Initial Lactate (mmol/L) & $1.3 \pm 0.7$ & $2.1 \pm 3.0$ & 0.48 \\
\hline AV-O2 Increase (\%) & $11.7 \pm 10.1$ & $6.8 \pm 7.9$ & $\mathbf{0 . 0 7}, 1.00$ \\
\hline Shortening Fraction (\%) & $19.7 \pm 6.5$ & $29.3 \pm 20.9$ & 0.32 \\
\hline Ejection Fraction (\%) & $35.1 \pm 17.2$ & $32.9 \pm 21.6$ & 0.80 \\
\hline Atrioventricular Valve Regurgitation & $1.9 \pm 1.1$ & $1.3 \pm 1.4$ & 0.30 \\
\hline Velocity-Time-Integral Increase (m) & $0.0111 \pm 0.283$ & $0.0250 \pm 0.269$ & $0.35, \mathbf{0 . 0 3}$ \\
\hline GLS (\%) & $-6.7 \pm 3.4$ & $-5.9 \pm 3.2$ & 0.58 \\
\hline GCS (\%) & $-5.2 \pm 1.5$ & $-7.3 \pm 4.0$ & 0.27 \\
\hline GLSR (1/sec) & $-0.74 \pm 0.40$ & $-0.53 \pm 0.26$ & 0.21 \\
\hline GCSR (1/sec) & $-0.57 \pm 0.18$ & $-0.65 \pm 0.34$ & 0.55 \\
\hline
\end{tabular}

Rows with two p-values compare the variable at full and minimal/no ECMO flow for each group. Echocardiogram measurements in this table were at full ECMO flow.

GLS - global longitudinal strain, GCS - global circumferential strain, GLSR - global longitudinal strain rate, GCSR - global circumferential strain rate

All bolded p-values are significant or near significant after Bonferonni correction. 